\title{
EFICÁCIA DOS SISTEMAS DEFENSIVOS EM SUPERIORIDADE E IGUALDADE \\ NUMÉRICA NO HANDEBOL: PANORAMA DE UMA COMPETIÇÃO EUROPEIA
}

\author{
EFFICACY OF DEFENSIVE SYSTEMS IN SUPERIORITY AND NUMERICAL EQUALITY \\ IN HANDBALL: PANORAMA OF A EUROPEAN COMPETITION
}

\section{EFICACIA DE LOS SISTEMAS DEFENSIVOS EN SUPERIORIDAD E IGUALDAD NUMÉRICA EN BALONMANO: PANORAMA DE UNA COMPETICIÓN EUROPEA}

\author{
Caio Petroni de Senzi Barreira ${ }^{1}$, Vinicius da Silva Musa ${ }^{1}$, Márcio Pereira Morato ${ }^{1}$, Rafael \\ Pombo Menezes 1 \\ caio.barreira@usp.br; vinicius.musa11@gmail.com; mpmorato@usp.br; \\ rafaelpombo@usp.br \\ ${ }^{1}$ Universidade de São Paulo, São Paulo, Brasil
}

Envío original: 2021-01-28 Reenviado: 2021-06-15 Aceptado: 2021-09-22

Publicado: 2021-10-07

Doi: https://doi.org/10.15517/pensarmov.v19i2.45584

\begin{abstract}
RESUMO
As ações e escolhas realizadas durante a fase defensiva no handebol podem ser determinantes para os resultados das partidas. Análises abordando essa fase do jogo vêm sendo realizadas para identificar aspectos determinantes e que possam auxiliar no planejamento e treinamentos das equipes. Este estudo teve como objetivo analisar a influência das relações numéricas nos sistemas defensivos fechados e abertos no handebol de alto nível. Para isso foram analisados 12 jogos do campeonato europeu de handebol de clubes, cujo instrumento de análise permitiu identificar a relação numérica do jogo (superioridade ou igualdade numérica defensiva) e quantificar os resultados (GO: gol; NG: não-gol; PP: perda de posse da bola) e os locais em que se encerraram as ações ofensivas. Foi utilizado o teste qui-quadrado para análise dos dados. Os resultados apontaram semelhanças para a conclusão do ataque mediante situações de igualdade com a utilização dos sistemas fechados ( $\mathrm{GO}=47,3 \% ; \mathrm{NG}=33,6 \% ; \mathrm{PP}=19,1 \%$ ) e dos sistemas abertos ( $G O=46,0 \% ; N G=34,3 \% ; P P=19,7 \%$ ). Para essa situação, demonstrou-se que a utilização dos sistemas fechados provoca o encerramento da posse mais distante do gol $(9 \mathrm{~m}-=56,9 \%$; $9 m+=43,1 \%)$, quando comparado à utilização dos sistemas abertos $(9 m-=64,5 \%$;
\end{abstract}


$9 m+=35,5 \%)$. Em situações de superioridade numérica defensiva a utilização dos sistemas abertos $(\mathrm{GO}=28,6 \%$; $\mathrm{NG}=53,6 \% ; \mathrm{PP}=17,9 \%$ ), quando comparados aos sistemas fechados ( $G O=49,3 \% ; N G=29,6 \% ; P P=21,1 \%$ ), apresentou maior eficácia nos resultados das ações. Além disso, as sequências se encerraram mais distantes do gol quando foram utilizados sistemas abertos $(9 \mathrm{~m}-=42,9 \% ; 9 \mathrm{~m}+=57,1 \%)$ em comparação aos sistemas fechados (9m$=68,3 \% ; 9 m+=31,7 \%$ ). Conclui-se que a escolha do sistema defensivo deve ser pautada no modelo de jogo da equipe e nas relações recorrentes do jogo, nos momentos de igualdade numérica (mais frequentes). Porém, em momentos de vantagem numérica defensiva limitar o espaço e o tempo para que os atacantes tomem decisões mostra-se como uma estratégia eficaz.

Palavras-chave: esportes coletivos, handebol, estratégias defensivas, Campeonato Europeu de Handebol.

\section{ABSTRACT}

The actions and choices made during the defensive phase in handball can be decisive for the results of the matches, and the analysis in this phase allows to identify determinant aspects that can assist in the planning and training of the teams. The aim of this work was to analyze the influence of numerical relations on closed and open defensive systems in high-level handball matches. Twelve matches of the European Handball club championship were analyzed, whose instrument of analysis allowed to identify the numerical relationship of the game (defensive superiority or equality) and to quantify the results (GO: goal; NG: non-goal; PP: loss of ball possession) and regions of the court in which the offensive actions ended. The chi-square test was used for data analysis. The results demonstrates similarities for the attack's conclusion in numerical equality situations, when defense used closed systems ( $G O=47,3 \% ; \quad N G=33,6 \% ; \quad P P=19,1 \%)$ and opened systems (GO=46,0\%; NG=34,3\%; $\mathrm{PP}=19,7 \%$ ). For this situation, it demonstrated that usage of closed systems stimulates the sequence end more distant from the goal $(9 \mathrm{~m}-=56,9 \% ; 9 m+=43,1 \%)$, when compared to opened systems usage $(9 \mathrm{~m}-=64,5 \% ; 9 \mathrm{~m}+=35,5 \%)$. In defensive numerical superiority the opened systems usage ( $\mathrm{GO}=28,6 \%$; $\mathrm{NG}=53,6 \% ; \mathrm{PP}=17,9 \%)$, compared to closed systems usage $(\mathrm{GO}=49,3 \% ; \mathrm{NG}=29,6 \% ; \mathrm{PP}=21,1 \%)$, presents higher efficacy in actions results. Otherwise, the sequences ends more distant from the goal when defense uses opened systems $(9 \mathrm{~m}-=42,9 \% ; 9 m+=57,1 \%)$, instead of the closed systems $(9 m-=68,3 \% ; 9 m+=31,7 \%)$. It was concluded that the choice of the defensive system should be based on the team's game model and in the game relationships, especially in situations of numerical equality (more frequent in games). However, when in defensive numerical advantage, limiting the space and time for attackers to make decisions seems to be an effective strategy. 
Keywords: team sports, handball, defensive strategies, European handball championship.

\section{RESUMEN}

Las acciones realizadas durante la fase defensiva en balonmano pueden ser determinantes para los resultados de los partidos, y los análisis en esta fase permiten identificar aspectos determinantes que pueden ayudar en la planificación y entrenamiento de los equipos. El objetivo de este trabajo fue analizar la influencia de las relaciones numéricas en sistemas defensivos cerrados y abiertos en partidos de balonmano de alto nivel. Se analizaron doce partidos del Campeonato de Europa de clubes de Balonmano, cuyo instrumento de análisis permitió identificar la relación numérica del juego (superioridad defensiva o igualdad) y cuantificar los resultados (GO: gol; NG: sin gol; PP: perdida del balón) y regiones de la cancha en las que terminaron las acciones ofensivas. Se utilizó la chi-cuadrado para el análisis de datos. Los resultados mostraron similitudes para la realización del ataque a través de situaciones de igualdad con el uso de sistemas cerrados $(\mathrm{GO}=47,3 \%$; $\mathrm{NG}=33,6 \%$; $\mathrm{PP}=$ $19,1 \%$ ) y sistemas abiertos ( $G O=46,0 \%$; NG $=34,3 \%$; PP = 19,7\%). Para esta situación, se demostró que el uso de sistemas cerrados provoca la pérdida del balón más leja de la portería $(9 \mathrm{~m}-=56,9 \% ; 9 \mathrm{~m}+=43,1 \%)$, cuando se compara con el uso de sistemas abiertos $(9 \mathrm{~m}-=$ $64,5 \% ; 9 \mathrm{~m}+=35,5 \%$ ). En superioridad numérica defensiva, el uso de sistemas abiertos (GO = 28,6\%; NG = 53,6\%; PP = 17,9\%), en comparación con sistemas cerrados $(\mathrm{GO}=49,3 \%$; $\mathrm{NG}=29,6 \%$; PP $=21,1 \%$, mostró mayor efectividad en los resultados de las acciones. Además, las secuencias terminaron más lejos del objetivo cuando se utilizaron sistemas abiertos $(9 \mathrm{~m}-=42,9 \% ; 9 m+=57,1 \%)$, en comparación con sistemas cerrados $(9 \mathrm{~m}-=68,3 \%$; $9 \mathrm{~m}+=31,7 \%)$. Se concluyó que la elección del sistema defensivo debe basarse en el modelo de juego del equipo y en las relaciones recurrentes del juego, especialmente en situaciones de igualdad numérica (más frecuentes). Sin embargo, cuando se tiene una ventaja numérica defensiva, limitar el espacio y el tiempo para que los atacantes tomen decisiones parece ser una estrategia eficaz.

Palabras clave: deportes colectivos, balonmano, estrategias defensivas, Campeonato Europeo de Balonmano 


\section{INTRODUÇÃO}

O handebol é caracterizado por um contexto que exige a resolução de situaçõesproblema decorrentes das interações de cooperação e oposição (Garganta, 1998; Menezes \& Reis, 2010). Diante dessas interações, as regras de ação ocupam posição central nas tomadas de decisão dos jogadores em cada fase do jogo (Gréhaigne, Bouthier \& David, 1997). As interações entre os jogadores associadas às restrições impostas pela regra do esporte caracterizam a lógica interna das diferentes modalidades, de acordo com as fases do jogo (Morato, Gomes, \& Almeida, 2012; Parlebas, 2001).

Durante a fase defensiva os jogadores tentam recuperar a posse da bola, dificultar a progressão do adversário e impedir a finalização (Bayer, 1994). No handebol, durante a fase de ataque posicionado, os defensores ocupam postos específicos (relacionados à estrutura dos sistemas ofensivos e defensivos) e ajustam seus comportamentos de acordo com o sistema utilizado (Menezes, 2011). Portanto, sabe-se que os sistemas defensivos zonais (fechados e abertos) possuem características particulares (Seco, 2005), as quais podem contribuir ou prejudicar o desempenho em determinadas situações do jogo. A escolha dos sistemas deve estar alinhada ao modelo de jogo da equipe, considerando as características técnicas, táticas e físicas de seus jogadores (Mendes, Greco, Ibáñez \& Nascimento, 2021).

Treinadores e pesquisadores vêm utilizando a quantificação de indicadores prédeterminados para analisar as ações mais frequentes nos jogos (Tabben et al., 2019), além das demandas técnicas, táticas e/ou fisiológicas (Hassan, Schrapf, \& Tilp, 2017; Michalsik, Aagaard, \& Madsen, 2015). Essas análises permitem o registro de aspectos relacionados ao desempenho esportivo, às ações dos jogadores e aos eventos críticos do jogo (Hughes \& Franks, 2015). O mapeamento desses parâmetros auxilia na identificação das variáveis relacionadas à performance das equipes e possibilitam maior entendimento sobre o jogo (Ferrari, Santos, \& Vaz, 2014; Meletakos, Vagenas, \& Bayios, 2011; Menezes \& Reis, 2010).

Por meio da análise da fase defensiva no handebol verificou-se que independentemente da ação realizada pelos defensores (faltas defensivas ou tentativas de recuperar a bola, por exemplo), as sequências ofensivas encerraram-se com mais frequência por meio de um arremesso ao gol (Fasold \& Redlich, 2018). Porém, as equipes vencedoras apresentaram maior eficácia na execução de elementos defensivos, quando buscaram provocar erros dos atacantes adversários (Rogulj, 2000). Nesse sentido, identificou-se que equipes com comportamentos mais agressivos e antecipativos na defesa podem provocar erros ofensivos com maior frequência (Gómez, Lago-Peñas, Viaño, \& González-Garcia, 2014). De maneira análoga, a eficácia do goleiro também pode ser apontada como fator preponderante para o rendimento das equipes na fase defensiva (Gutiérrez-Davila, Rojas, Ortega, Campos, \& Parraga, 2011). 
A partir desse panorama, a identificação dos indicadores de performance (como recuperação da bola, erros ofensivos e regiões de eficácia defensiva) oferece informações aos treinadores, permite avaliar o modelo de jogo da equipe e subsidiar a escolha do sistema defensivo. Apesar do conhecimento dos indicadores de performance relacionados à fase defensiva, nota-se ainda uma lacuna referente à comparação dos resultados das sequências defensivas diante de diferentes situações numéricas no jogo. Nesse sentido, emergem questões como: a) "De que maneira a utilização dos sistemas defensivos influencia no jogo de handebol?"; b) "Quais os resultados mais recorrentes nas diferentes relações numéricas ao considerar os sistemas defensivos?"; e c) "Em quais regiões da quadra as ações se encerram essas situações?". Pautando-se nessas perguntas, esse estudo teve como objetivo identificar a influência da utilização dos sistemas defensivos nas relações numéricas de igualdade e superioridade numérica defensiva. Especificamente, buscou-se analisar as ações mais recorrentes nas relações numéricas mediante diferentes sistemas defensivos e onde essas ocorreram.

\section{MÉTODO}

Este estudo partiu de uma abordagem de observação sistemática sem participação no contexto do jogo. Esse método é constituído pela observação de jogos, no qual o observador é responsável por delimitar os parâmetros a serem quantificados e, posteriormente, analisar e interpretar os dados provenientes das observações (Wright, Carling, \& Collins, 2014).

\section{Amostra}

Foram analisados 12 jogos do Campeonato Europeu masculino de handebol de clubes (Champions League) da temporada 2015/2016 (quartas-de-finais - 8 jogos; semi-finais - 2 jogos; disputa de $3^{\mathrm{a}}$ e $4^{\mathrm{a}}$ colocação - 1 jogo; e final - 1 jogo), que resultaram em 988 sequências ofensivas. A amostra representa o mais alto nível de desempenho da modalidade na referida competição, como forma de garantir o controle da qualidade de oposição (Marcelino, Sampaio, \& Mesquita, 2011), uma vez que as equipes europeias possuem hegemonia no cenário do handebol (maior potencial financeiro e presença de jogadores que compõem as seleções nacionais de países de fora da Europa, inclusive).

As imagens utilizadas para a análise foram obtidas mediante consulta ao site com política de acesso/domínio público: laola1.tv e por envolver seres humanos (embora sem a intenção de identificá-los), o trabalho foi submetido e aprovado pelo Comitê de Ética em Pesquisa da Escola de Educação Física e Esporte de Ribeirão Preto (CAAE: 67387017.7.0000.5659, parecer 2.095.778). 


\section{Protocolo de observação}

De forma a padronizar as análises e devido à familiaridade dos pesquisadores com o software, os jogos foram observados por meio do software livre Kinovea $($ v.0.8.15 e os dados computados em planilha eletrônica ad hoc, armazenadas no Google Drive ${ }^{\circ}$. Todo o protocolo de análise baseou-se em indicadores de performance (Hughes \& Bartlett, 2002) durante o jogo posicionado (excluindo-se, portanto, as transições) para identificar os resultados das ações ofensivas, a posse da bola, o sistema defensivo, a relação numérica (atacantes vs. defensores) e a região da quadra onde o ataque terminou. Para as relações numéricas foram analisadas apenas situações de superioridade e igualdade, uma vez que em inferioridade numérica as equipes utilizaram-se apenas dos sistemas defensivos fechados. A Tabela 1 apresenta as categorias de análise, os indicadores de performance utilizados nas análises, seguido de suas descrições.

Tabela 1

Variáveis observadas durante as análises dos jogos

\begin{tabular}{|c|c|c|}
\hline & Variável & Descrição \\
\hline \multirow{2}{*}{$\begin{array}{l}\text { Sequências } \\
\text { ofensivas com } \\
\text { finalização }\end{array}$} & Gol (GO) & Gol anotado pela equipe atacante. \\
\hline & Não Gol (NG) & $\begin{array}{l}\text { Defesa do goleiro, bloqueio do defensor quando, } \\
\text { arremesso na trave ou arremesso para fora. }\end{array}$ \\
\hline $\begin{array}{l}\text { Sequências } \\
\text { ofensivas sem } \\
\text { finalização }\end{array}$ & $\begin{array}{l}\text { Perda da posse } \\
\text { sem finalização } \\
(P P)\end{array}$ & $\begin{array}{l}\text { Quando o defensor retoma o domínio da bola } \\
\text { (desarme), interceptação de passe, violação da regra } \\
\text { por um atacante ou qualquer outro fato que provocou } \\
\text { a perda da posse da bola. }\end{array}$ \\
\hline \multirow{2}{*}{$\begin{array}{l}\text { Sistemas } \\
\text { defensivos }\end{array}$} & Fechados & $\begin{array}{l}\text { Defensores posicionados próximos à área de gol e } \\
\text { em uma única linha }(6: 0) \text {. }\end{array}$ \\
\hline & Abertos & $\begin{array}{l}\text { Defensores posicionados em duas ou mais linhas } \\
(5: 1,4: 2,3: 3,3: 2: 1, \ldots)\end{array}$ \\
\hline \multirow{2}{*}{$\begin{array}{l}\text { Relação } \\
\text { numérica }\end{array}$} & $\begin{array}{l}\text { Igualdade } \\
\text { numérica }\end{array}$ & $\begin{array}{l}\text { Considerou-se igualdade numérica quando ao } \\
\text { término de uma sequência, ambas as equipes } \\
\text { encontravam-se com o mesmo número de jogadores } \\
\text { em quadra. }\end{array}$ \\
\hline & $\begin{array}{l}\text { Superioridade } \\
\text { numérica } \\
\text { defensiva }\end{array}$ & $\begin{array}{l}\text { Considerou-se superioridade numérica defensiva } \\
\text { quando ao término de uma sequência, a equipe em } \\
\text { fase ofensiva encontrava-se com um ou mais } \\
\text { jogadores punidos por dois minutos. }\end{array}$ \\
\hline
\end{tabular}

Fonte: os autores. 
Para a análise do local da quadra em que se encerraram as sequências foram anotadas as regiões mais distantes do alvo (para trás da linha que delimita os 9 metros) e as mais próximas (para frente da linha que delimita os 9 metros).

\section{Confiabilidade e análise dos dados}

Para garantir a replicabilidade do estudo e a confiabilidade dos dados, dois observadores com experiências prévias como jogadores, treinadores e pesquisadores da área do handebol participaram das análises dos jogos e dos dados obtidos. Primeiramente realizou-se a uniformização (concordância por consenso) e aprendizagem dos critérios, seguido por um treinamento inicial para a coleta dos dados (Anguera, 1999; Anguera \& Hernández, 2013). Após o primeiro contato com as análises verificou-se a consistência dos resultados obtidos após nova observação de um mesmo indivíduo (fidedignidade) e de dois indivíduos diferentes (objetividade) (Thomas, Nelson, \& Silverman, 2012), sendo a segunda realizada após 15 dias de terminada a primeira.

A verificação da confiabilidade intraobservador e interobservador ocorreu com $30 \%$ da amostra (Fleiss, Levin \& Paik, 2013). A confiabilidade intra e interobservadores foi testada pelo índice Kappa de Cohen, que exclui a possibilidade de existirem acordos por acaso. Os índices de confiabilidade intraobservador $(0,985$ a 1,00 para observador 1 e 0,900 a 0,985 para observador 2$)$ e interobservador $(0,865$ à 0,996$)$ atingiram valores que apontam boa concordância para a análise (Anguera \& Hernández, 2013).

Os resultados foram estruturados a partir de dois grupos: situações de igualdade numérica e situações de superioridade numérica defensiva. Dessa forma, utilizou-se do teste de qui-quadrado para comparar a homogeneidade dos indicadores (Gol, Não Gol, Perda da Posse) frente aos sistemas defensivos analisados (fechado e abertos). Em seguida foi comparada a homogeneidade dos indicadores referentes ao local da quadra frente aos sistemas defensivos. Para todos os testes o nível de significância foi fixado em $p<0,05$. Os dados obtidos por meio das análises realizadas permitiram o agrupamento das variáveis observadas e o cálculo de variáveis pautado na estatística descritiva. Tal quantificação permitiu a análise das situações de jogo posicionado no handebol, possibilitando a diferenciação entre as consequências da utilização de sistemas defensivos em diferentes relações numéricas, além das regiões com maior incidência de encerramento destas sequências ofensivas.

\section{RESULTADOS}

De forma geral, a utilização dos sistemas defensivos demonstraram resultados similares nas situações de igualdade numérica, porém apresentaram diferenças nas situações de superioridade defensiva. Notou-se uma tendência das sequências ofensivas serem finalizadas 
ao gol independente do sistema defensivo utilizado, porém maior eficácia para os sistemas abertos em situações de superioridade numérica. Já os locais da quadra em que as ações se encerraram, apresentaram diferenças para todas as situações analisadas.

Diante de ambos os sistemas defensivos em situação de igualdade numérica, a frequência dos gols foi superior aos demais índices, enquanto a troca da posse da bola sem finalização apresentou menor frequência em ambos os contextos. No entanto, não foi encontrada diferença significativa na comparação entre os sistemas defensivos $X^{2}(1)=0.08$, $p=0,1$. As situações de superioridade numérica defensiva revelaram que os sistemas fechados deixaram as equipes mais propensas a sofrerem gols. Já os sistemas defensivos abertos apresentaram maior índice de troca da posse da bola (sem sofrer gol), que pode ser verificada por meio da diferença significativa entre o uso dos sistemas, $X^{2}(1)=6, p=0,04$. Na tabela 2, estão apresentados os resultados das ações diante da utilização dos sistemas defensivos nas diferentes relações numéricas.

\section{Tabela 2}

Resultados das ações ofensivas em diferentes contextos defensivos (GO: gol; NG: não gol; PP: perda da posse sem finalização)

\begin{tabular}{ccccc}
\hline Relação numérica & Sistemas defensivos & GO & NG & PP \\
\hline Igualdade $(\mathbf{n = 8 1 8})$ & Fechados $(n=681)$ & $47,3 \%$ & $33,6 \%$ & $19,1 \%$ \\
Superioridade & Fechados $(n=142)$ & $49,3 \%$ & $29,6 \%$ & $21,1 \%$ \\
defensiva (n=170) & Abertos $(n=28)$ & $28,6 \%$ & $53,6 \%$ & $17,9 \%$ \\
\hline
\end{tabular}

Fonte: os autores.

Em relação às regiões da quadra onde foram finalizadas as sequências, observou-se que em situações de igualdade numérica houve maior frequência de finalização em regiões mais próximas ao alvo, em ambos os sistemas defensivos analisados. Foi possível notar ainda que diante de sistemas defensivos abertos, os ataques tendem a se encerrar mais próximos ao gol do que mediante sistemas fechados, embora não tenha sido encontrada diferença significativa na comparação entre sistemas $X^{2}=2, p=0,1$. Já a superioridade numérica defensiva apresentou diferença significativa na utilização dos sistemas $X^{2}=6, p=0,02$, de modo que a utilização dos sistemas defensivos fechados permitiu, além da maior frequência de gols, os arremessos mais próximos do gol. Por outro lado, os sistemas defensivos abertos tiveram menor frequência de gols, com as sequências ofensivas encerradas mais distantes 
do alvo. Na Figura 2 estão apresentadas as regiões da quadra onde foram finalizadas as sequências ofensivas nos diferentes contextos analisados.
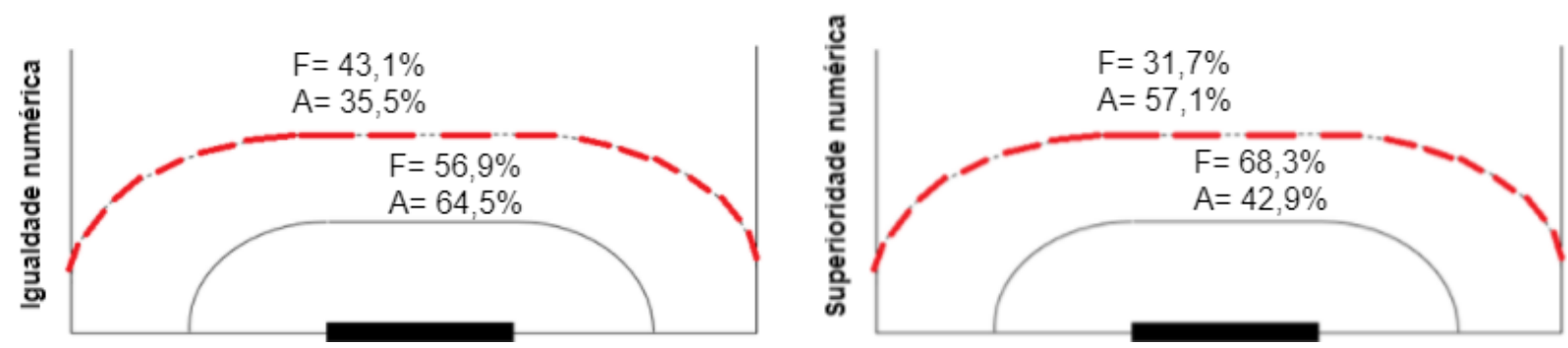

Figura 2. Regiões da quadra nas quais foram finalizados os processos ofensivos diante de sistemas defensivos fechados $(F)$ e abertos $(A)$ em igualdade e superioridade numérica. Fonte: os autores.

\section{DISCUSSÃO}

Este estudo teve como objetivo analisar as ações mais recorrentes nas condições de igualdade e superioridade numérica defensiva mediante diferentes sistemas de jogo, assim como onde essas ocorreram. Com base nos resultados destacou-se a necessidade de adaptação da escolha dos sistemas defensivos, de acordo com os diversos fatores que interferem no jogo. Apontou-se maior diferença na utilização dos sistemas defensivos em situações de superioridade numérica, com maior eficácia para os sistemas abertos. E os locais de encerramento das sequências reforçaram a importância da pressão defensiva e da ocupação espacial, principalmente nas situações de superioridade numérica.

Em situações de igualdade numérica os resultados apontaram que independentemente do sistema defensivo utilizado há maior frequência na ocorrência de gols quando comparado às ações que não resultaram em gol ou proporcionaram a perda da posse da bola. Esses achados demonstram que o desempenho defensivo em igualdade numérica pode estar mais relacionado com a sincronia entre os defensores e o goleiro (Gutiérrez-Davila et al., 2011; Hansen et al., 2017) para as tomadas de decisão e para a recuperação da posse de bola sem sofrer o gol (Gómez et al., 2014; Jiménez, Morillo Baro, Reigal Garrido, Morales Sánchez, \& Hernández-Mendo, 2020), do que com a escolha propriamente dita dos sistemas defensivos a serem utilizados.

A semelhança entre os resultados das ações em igualdade numérica combinado ao fato da escolha dos sistemas defensivos otimizar a utilização de meios técnico-táticos individuais e coletivos (Mendes et al., 2021), reforça a importância do desenvolvimento das relações de cooperação entre os defensores e dos defensores com o goleiro. Essas escolhas devem pautar-se no modelo de jogo de suas equipes, considerando as características dos jogadores e as relações (cooperação e oposição) recorrentes do jogo (Mendes et al., 2021), que 
subsidiam o processo de tomada de decisão. Além disso, essa escolha ainda pode proporcionar melhor compreensão das tarefas atribuídas a cada defensor na fase defensiva, diante das relações e espaços oriundos das situações do jogo (Menezes, Reis \& Tourinho, $\underline{2015})$.

No que condiz ao local da quadra que se encerra as ações em igualdade numérica, demonstrou-se uma tendência para as regiões mais distantes do gol diante dos sistemas defensivos fechados e mais próximas nos sistemas abertos. Esses achados também devem ser considerados no momento da escolha dos sistemas defensivos a serem utilizados, uma vez que ressaltam suas características. Os sistemas defensivos fechados têm como característica a proteção da região mais próxima do gol, a proximidade entre os jogadores, a ocupação da quadra em largura e a menor necessidade de longos deslocamentos (Seco, 2005). Em contrapartida, os sistemas defensivos abertos são caracterizados por uma ocupação da quadra com maior distância entre os jogadores e com maior profundidade, em detrimento da proteção das regiões mais próximas do gol (Seco, 2005). Assim sendo, foi notória a busca das equipes para a exploração dessas possíveis limitações dos tipos de sistemas defensivos.

Considerando as situações de superioridade numérica defensiva, o uso dos sistemas defensivos abertos se mostrou mais eficaz, diferentemente do observado em situações de igualdade numérica. Esse fato demonstra que a escolha dos sistemas defensivos quando a equipe encontra-se em superioridade numérica influencia os resultados das ações e o cumprimento dos objetivos pelos atacantes adversários. A eficácia na utilização dos sistemas defensivos abertos (em superioridade numérica) pode estar relacionada à constante perturbação aos atacantes e à ruptura do ritmo ofensivo, que dificulta o acesso às regiões mais próximas do gol e aumenta a pressão espaço-temporal para a tomada de decisão dos atacantes (Fernández Romero, Casais Martínez, Vila Suárez, \& Cancela Carral, 1999; Menezes, 2011).

Além disso, as relações mais diretas entre atacantes e defensores, proporcionadas pelo uso desse sistema (Seco, 2005), também podem ter colaborado com as combinações entre os defensores e o goleiro. Esses achados corroboram os estudos que analisaram a execução dos elementos defensivos e apontaram maior eficácia para as situações em que os defensores tentaram induzir os atacantes ao erro (Rogulj, 2000).

Os resultados relacionados aos locais em que se encerraram as ações ofensivas ainda reforçaram as questões inerentes à pressão defensiva e ao acesso às regiões mais próximas do gol. Nos momentos em que foram utilizados sistemas defensivos abertos as ações encerraram-se mais distantes do alvo em comparação aos sistemas fechados. Esse fato corrobora a característica dos sistemas abertos de se contrapor aos atacantes com maior profundidade, obrigando-os a realizar suas ações mais distantes do alvo (Fernández Romero 
et al., 1999; Seco, 2005; Menezes, 2011), e revela, ainda, um contexto profícuo para a sua utilização em situações de superioridade numérica defensiva.

\section{CONCLUSÃO}

O presente estudo analisou a utilização dos sistemas defensivos em diferentes situações do jogo de handebol. Os achados revelaram que em situações de igualdade numérica a escolha dos sistemas defensivos pode não alterar os resultados das ações, devendo pautar-se, portanto, nas diferentes possibilidades que os jogadores e as equipes apresentam para o jogo. Nas situações de superioridade numérica defensiva, os sistemas defensivos abertos demonstraram maior eficácia do que os fechados. Esse fato ressalta a importância da ocupação espacial e da pressão dos defensores sobre os atacantes para o êxito defensivo.

Por fim, este estudo pode contribuir para o contexto do handebol no que tange à escolha dos sistemas defensivos em função de situações específicas do jogo (como igualdade e superioridade numérica). Ao contribuir para as reflexões e escolhas dos treinadores, os apontamentos deste estudo podem subsidiar a construção dos modelos de jogo das equipes em diferentes categorias, bem como para o planejamento dos treinamentos, especialmente em relação aos aspectos estruturais e de dinâmica dos sistemas defensivos. Em contrapartida, para as análises deste estudo foram observados apenas os dados do alto rendimento que consideraram apenas a classificação em regiões próximas ou distantes do gol. Esses aspectos revelam que esforços futuros possam ser direcionados para um número maior de regiões da quadra e para os diferentes contextos do handebol (outras categorias ou países), contribuindo para o aprofundamento das discussões inerentes a esta temática.

\section{REFERÊNCIAS}

Anguera, M. T. (1999). Observación en deporte y conducta cinésico-motriz: aplicaciones. Barcelona: Edicions Universitat Barcelona.

Anguera, M. T., \& Hernández, A. (2013). La metodología observacional en el ámbito del deporte. E-balonmano.com: Revista de Ciencias del Deporte, 9(3), 135-160. Recuperado de https://e-balonmano.com/ojs/index.php/revista/article/view/139

Bayer, C. (1994). O ensino dos desportos colectivos. Lisboa: Dinalivros.

Fasold, F., \& Redlich, D. (2018). Foul or no foul? Effects of permitted fouls on the defence performance in team handball. Journal of Human Kinetics, 63(1), 53-59. doi: https://doi.org/10.2478/hukin-2018-0006

Fernández Romero, J. J., Casais Martínez, L., Vila Suárez, H., \& Cancela Carral, J. M. (1999). Balonmán: manual básico. Santiago: Edicións Lea. 
Ferrari, W. R., Santos, J. V., \& Vaz, V. P. S. (2014). Offensive process analysis in handball: Identification of game actions that differentiate winning from losing teams. American Journal of Sports Science, 2(4), 92-96. Recuperado de http://www.sciencepublishinggroup.com/journal/paperinfo. aspx?journalid=155\&doi=1 $\underline{0.11648 / j . a j s s .20140204 .14}$

Fleiss, J. L., Levin, B., \& Paik, M. C. (2013). Statistical methods for rates and proportions (3 $3^{\text {rd }}$ ed.). New Jersey: John Wiley \& Sons.

Garganta, J. (1998). Para uma teoria dos jogos desportivos colectivos. In A. Graça \& J. Oliveira (Eds.), O ensino dos jogos desportivos (pp. 11-26). Porto: Universidade do Porto.

Gómez, M. Á., Lago-Peñas, C., Viaño, J., \& González-Garcia, I. (2014). Effects of game location, team quality and final outcome on game-related statistics in professional handball close games. Kinesiology, 46(2), 249-257. Recuperado de https://bit.ly/3uacdWv

Gréhaigne, J. F., Bouthier, D., \& David, B. (1997). Dynamic-system analysis of opponent relationships in collective actions in soccer. Journal of Sports Sciences, 15(2), $137-$ 149. doi: https://doi.org/10.1080/026404197367416

Gutiérrez-Davila, M., Rojas, J., Ortega, M., Campos, J., \& Parraga, J. (2011). Anticipatory strategies of team-handball goalkeepers. Journal of Sports Sciences, 29(12), 13211328. doi: https://doi.org/10.1080/02640414.2011.591421

Hansen, C., Sanz-Lopez, F., Whiteley, R., Popovic, N., Ahmed, H. A., \& Cardinale, M. (2017). Performance analysis of male handball goalkeepers at the World Handball Championship 2015. Biology of Sport, 34(4), 393-400. doi: https://doi.org/10.5114/biolsport.2017.69828

Hassan, A., Schrapf, N., \& Tilp, M. (2017). The prediction of action positions in team handball by non-linear hybrid neural networks. International Journal of Performance Analysis in Sport, 17(3), 293-302. doi: http://dx.doi.org/10.1080/24748668.2017.1336688

Hughes, M., \& Bartlett, R. M. (2002). The use of performance indicators in performance analysis. Journal of Sports Sciences, 20(10), 739-754. doi: https://doi.org/10.1080/026404102320675602

Hughes, M., \& Franks, I. (2015). Essentials of performance analysis in sport. Choice reviews online. London: Routledge.

Salas Jiménez, J., Morillo Baro, J., Reigal Garrido, R., Morales Sánchez, V., \& HernándezMendo, A. (2020). Polar Coordinate Analysis to Study Counterattacks in Senior and Under-16 Men's Handball. Cuadernos de Psicología del Deporte, 20(1), 48-61. doi: https://doi.org/10.6018/cpd.396521

Marcelino, R., Sampaio, J., \& Mesquita, I. (2011). Investigação centrada na análise do jogo: da modelação estática à modelação dinâmica. Revista Portuguesa de Ciências do 
Desporto,

11(1),

481-499.

Recuperado

de

https://rpcd.fade.up.pt/ arquivo/artigos soltos/2011-1/09.pdf

Meletakos, P., Vagenas, G., \& Bayios, I. (2011). A multivariate assessment of offensive performance indicators in men's handball: trends and differences in the World Championships. International Journal of Performance Analysis in Sport, 11(2), 285295. doi: https://doi.org/10.1080/24748668.2011.11868548

Mendes, J. C., Greco, P. J., Ibáñez, S. J., \& Nascimento, J. V. (2021). Construcción del modelo de juego en balonmano. Pensar en Movimiento: Revista de Ciencias del Ejercicio y la Slaud, 19(1), 1-25. doi: https://doi.org/10.15517/pensarmov.v19i1.42052

Menezes, R. P. (2011). Modelo de análise técnico-tática do jogo de handebol: necessidades perspectivas e implicações de um modelo de interpretação das situações de jogo em tempo real (Tese do Doutorado). Universidade Estadual de Campinas, Brasil. Recuperado de http://www.bibliotecadigital.unicamp.br/document/?code=000796445

Menezes, R. P., \& Reis, H. H. (2010). Análise do jogo de handebol como ferramenta para sua compreensão técnico-tática. Motriz-Revista De Educação Física, 16(2), 458-467. Recuperado de https://www.periodicos.rc.biblioteca.unesp.br/index.php/motriz/article/view/19806574.2010v16n2p458/3002

Menezes, R., Reis, H. H., \& Tourinho, H. (2015). Ensino-Aprendizagem-Treinamento dos elementos técnico-táticos defensivos individuais e coletivos nas categorias Infantil, Cadete e Juvenil, Movimento, 21(1), 261-273. doi: https://doi.org/10.22456/1982$\underline{8918.46159}$

Michalsik, L. B., Aagaard, P., \& Madsen, K. (2015). Technical activity profile and influence of body anthropometry on playing performance in female elite team handball. The Journal of Strength \& Conditioning Research, 29(4), 1126-1138. doi: https://doi.org/10.1519/jsc.0000000000000735

Morato, M., Gomes, M., \& Almeida, J. (2012). Os processos auto-organizacionais do goalball. Revista Brasileira de Ciências do Esporte, 34(3), 741-760. doi: https://doi.org/10.1590/S0101-32892012000300015

Parlebas, P. (2001). Juegos, deporte y sociedad: léxico de praxiología motriz. Barcelona: Editorial Paidotribo.

Rogulj, N. (2000). Differences in situation-related indicators of the handball game in relation to the achieved competitive results of teams at 1999 World Championship in Egypt. Kinesiology, 32(2), 63-74.

Seco, J. (2005). Conceptos de ataque frente a variantes defensivas 6:0 y 5:1. E-balonmano. com: Revista de Ciencias del Deporte, 1(1), 3-16. Recuperado de https://dialnet.unirioja.es/descarga/articulo/1371312.pdf 
Tabben, M., Landreau, P., Chamari, K., Juin, G., Ahmed, H., Farooq, A., Bahr, P., \& Popovic, N. (2019). Age, player position and 2 min suspensions were associated with match injuries during the 2017 Men's Handball World Championship (France). British Journal of Sports Medicine, 53(7), 436-441. doi: https://doi.org/10.1136/bjsports-2018-099350

Thomas, J. R., Nelson, J. K., \& Silverman, S. J. (2012). Métodos de pesquisa em atividade física ( $6^{\mathrm{a}}$ ed.). Porto Alegre: Artmed.

Wright, C., Carling, C., \& Collins, D. (2014). The wider context of performance analysis and it application in the football coaching process. International Journal of Performance Analysis in Sport, 14(3), 709-733. doi: https://doi.org/10.1080/24748668.2014.11868753 